\title{
Unified Non-Inverting and Inverting PWM AC-AC Converter with Versatile Modes of Operation
}

\author{
Peng Li, Member, IEEE, Yihua Hu, Senior Member, IEEE
}

\begin{abstract}
This paper proposes unified non-inverting and inverting AC-AC converter (UNI-AC) using pulse width modulation (PWM) for the utility voltage compensation. It offers four effective switching states to regulate the output voltage in bipolar manner, facilitating versatile modes of operation with different number of switching states being modulated. Each mode of the proposed UNI-AC is able to compensate both the grid voltage sag and swell problems due to its bipolar voltage gain. The operational principle and comparison for all these modes are investigated in details. Also, the UNI-AC is reversible and compatible with full range of power factor. Other technical merits offered by the proposed approach include the compact hardware installation, reduced switch voltage stress (also low $d v / d t$ ) and decreased control complexity. Detailed analysis and experimental verification are presented in this paper.
\end{abstract}

Index Terms-Bipolar voltage gain, PWM direct ac-ac converter, utility voltage compensation, versatile modes of operation.

\section{INTRODUCTION}

$\mathrm{O}$ WING to the rapidly increasing load condition diversity and various disturbance sources at the distribution level of power system, the grid voltages supplied to local consumers are exposed to an increasing risk of power quality problems such as utility voltage sag/swell, three-phase unbalance and voltage fluctuation (flicker) phenomenon, which are usually originated from the severe load variations, for example, the transient of large power motors; as well as the vast use and uneven spread of single-phase load $[1,2]$.

The flexible ac transmission system (FACTS) devices are a range of techniques to address the grid control issues. Among all kinds of FACTS devices, the series compensator is able to stabilize the distributive grid voltage during the power quality events, serving as the dynamic voltage restorer (DVR) [3-5].

Typical DVR employs the dc-ac voltage source converter

Manuscript received June 02, 2016; revised August 11, 2016; accepted September 13, 2016.

$\mathrm{P}$. Li is with the Electronic \& Electrical Engineering Department, University of Strathclyde, Glasgow, G1 1XW, U.K. (e-mail: peng.li@strath.ac.uk).

Y. Hu is with the Electrical Engineering and Electronics Department, University of Liverpool, Liverpool, L69 3GJ, U.K. (e-mail: y.hu35@liverpool.ac.uk).
(VSC) to generate the desired ac voltage in series with the grid and supply proper amount of power to the load. Specifically, to maintain the grid voltage amplitude without considering the harmonic distortion and phase-shift, the direct ac-ac converter is viable to replace the sizeable dc-ac solution by saving the dc-link capacitor bank; thus, reducing the footprint and cost as shown in Fig. 1. Under this motivation, the ac-ac converters for voltage amplitude regulation are of interest to be studied continuously $[6,7]$.

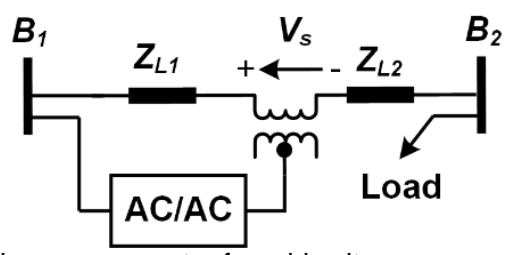

Fig. 1. DVR with ac-ac converter for grid voltage compensation.

The basic ac-ac converters including buck, boost and buck-boost topologies all suffer from a unipolar voltage gain range, which, in use of the grid voltage compensation, restricts them to mitigate either voltage sag or swell but not both. Although the tapped-winding transformer can assist them in generating bipolar voltage, the combined system cost and total volume are uneconomical [8].

In order to overcome this constraint, the Z-source topology based ac-ac converter has been presented to achieve the bipolar voltage output ability by an additional impedance network [9]. However, this converter has a discontinuous voltage transfer ratio; also its input and output ports are floating to each other, increasing the insulation demand for the coupling transformers in grid applications. An alternative version of Z-source ac-ac converter in [10] is able to share the same ground for input and output but still has a high cost due to the passive device network. In [11, 12], a variety of quasi-Z-source based ac-ac converters are reported with some advantages compared to the original Z-source ac-ac converter such as the reduced passive devices, improved input profile and common ground shared by input and output terminals. The Gamma structure Z-source ac-ac converter in [13] employs the coupled inductor to offer an extra hardware design degree of freedom for varying the voltage gain. The main constraint of the above solutions is their incapability of generating in-phase step-down ac voltage; and hereby, the extremely sharp change in its gain curve that is challenging for the adaptive adjustment of duty cycle command 
from the controller. A reversed scheme of [12] is analyzed in [14]. This solution has a continuous bipolar voltage transfer ratio with the use of the inner voltage boosting cell; besides, the ground sharing feature is kept. However, it still suffers from the high voltage and current stresses for the power switches (low device utilization) as in the Z-source type converters due to the voltage lifting capacitors; also, in these approaches, the high order passive elements in the impedance network result in a low bandwidth for the converter transfer functions; hence, slow dynamic response.

In an effort to reduce the passive device volume, a current source type composite ac-ac converter using six unidirectional switches is proposed in [15] with a wide range bipolar voltage generation capability. It uses less number of passive elements compared to the Z-source based schemes; therefore, reducing the voltage (current) stress on the semiconductor device and enhancing the converter dynamic response. The drawbacks of this solution include the lack of common ground sharing and the complex modulation due to its composite structure.

This paper investigates a unified non-inverting and inverting ac-ac converter (UNI-AC) operating in versatile modes thanks to the extra control degree of freedom. The UNI-AC offers following features: it achieves continuous and bipolar voltage transfer ratio without sharp changes as in Z-source based converters; it has improved flexibility and various operation modes with increased number of control variables; the use of less passive devices in the UNI-AC leads to a reduced footprint and higher switching device utilization (low electrical stresses); also, the common ground between the input and output ports is retained. The reminder of this paper is arranged as follows: section II describes the principles for different operation modes of the UNI-AC; in section III, the performance evaluation for the proposed converter under each mode is carried out; based on which, the design guidelines of the UNI-AC is supplied in section IV; then, in section $\mathrm{V}$, the experimental test results are presented to verify the proposed approach; finally, the conclusions are highlighted in section VI.

\section{Operational Principle of The Proposed UNI-AC}

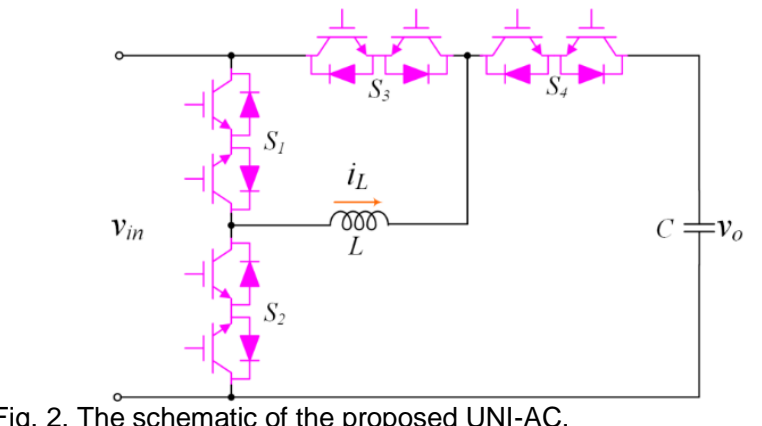

Fig. 2. The schematic of the proposed UNI-AC.

The proposed UNI-AC is depicted in Fig. 2, where the four bidirectional switches using the back-to-back series-connected insulated gate bipolar transistor (IGBT) are adopted to facilitate the bidirectional current conduction and bidirectional voltage blocking capability. In Fig. 2, the two switches in the same leg such as $S_{1}$ and $S_{2}$ (or $S_{3}$ and $S_{4}$ ) are complementarily triggered using pulse width modulation (PWM) scheme; and also, there must be two conducted switches to form a power path at each instance. Based on these constraints, it is concluded that there are four switching states are valid for regulating the output voltage as described in Fig. 3.

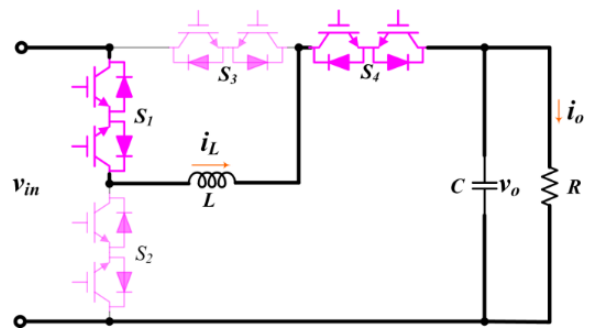

(a)

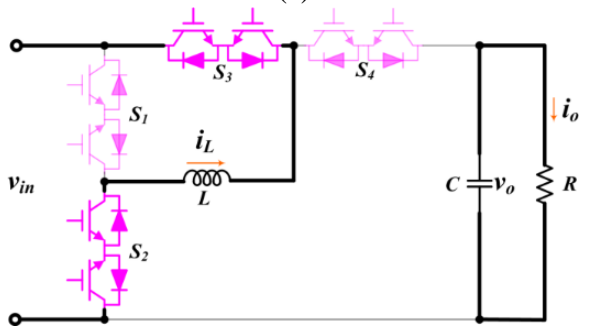

(b)

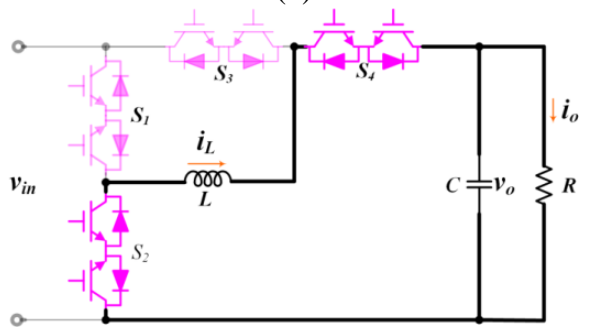

(c)

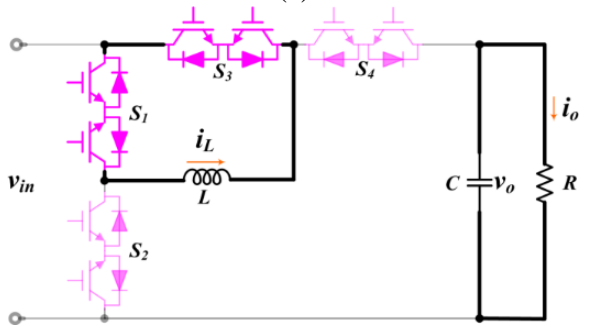

(d)

Fig. 3. Four effective switching states of UNI-AC: (a) state I with $S_{1}$ and $S_{4}$ turned on; (b) state II with $S_{2}$ and $S_{3}$ turned on; (c) state III with $S_{2}$ and $S_{4}$ turned on; (d) state IV with $S_{1}$ and $S_{3}$ turned on.

The switching state I in Fig. 3(a) turns on $S_{1}$ and $S_{4}$ to force the inductor $L$ to release energy for charging the capacitor $C$. While for switching state II in Fig. 3(b), $L$ is charged by the input source with $S_{3}$ and $S_{2}$ turned on; and the output current $i_{o}$ is directly drawn from the output capacitor $C$. In Fig. 3(c), $S_{4}$ and $S_{2}$ conduct to form a zero-input power loop between $L$ and $C$, which is noted as switching state III. The switching state IV when $S_{3}$ and $S_{1}$ are turned on as in in Fig. 3(d) represents the inductor current $i_{L}$ freewheeling mode with the output current supplied by capacitor $C$. Based on Fig. 3 , it is observed that different combinations of the four states can result in versatile modes of operation; and its control degrees of freedom varies accordingly. Detailed analysis for all possibilities is supplied in this section. 


\section{A. Operation mode $A$}

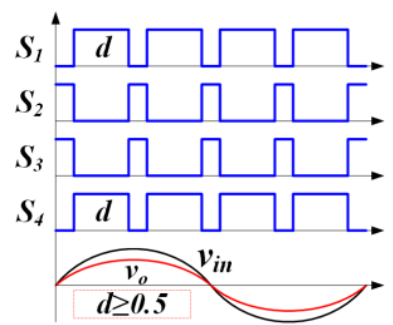

(a)

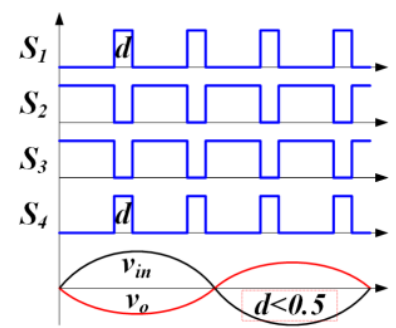

(b)
Fig. 4. Gate signals of the UNI-AC in operation mode A: (a) positive voltage gain; (b) negative voltage gain.

With the gate signals arranged as in Fig. 4, this mode uses the switching states I-II of Fig. 3 for the voltage conversion. The four switches are viewed to be separated into two groups $\left\{S_{1}\right.$, $\left.S_{4}\right\}$ and $\left\{S_{2}, S_{3}\right\}$; and these two groups are conducted in a complementary manner. Assuming the switching frequency is sufficiently high compared to the fundamental frequency, if $d$ is the duty cycle of $\left\{S_{1}, S_{4}\right\}$ in pulse width modulation (PWM), the switching average model of the UNI-AC is achieved as (1) using the established modelling procedures in $[16,17]$.

$$
\left\{\begin{array}{l}
L \cdot \frac{d i_{L}}{d t}=d \cdot\left(v_{i n}-v_{o}\right)-(1-d) \cdot v_{i n} \\
C \cdot \frac{d v_{o}}{d t}=d \cdot\left(i_{L}-i_{o}\right)-(1-d) \cdot i_{o}
\end{array}\right.
$$

In steady state, since all state variables of the UNI-AC are purely distributed in fundamental frequency, the right terms of (1) is approximately viewed as zero due to their low variation rates. Hence, the transfer ratio of the voltage amplitude from input voltage to output voltage is achieved based on a quasi-steady-state analysis as shown in (2), where $v_{o_{-} m}$ and $v_{i n \_m}$ are the magnitude of the output and input voltages respectively.

$$
M_{A}=\frac{v_{o_{-} m}}{v_{\text {in_m }_{-}}}=2-\frac{1}{d}
$$

Then, the plot for the relationship between $M_{A}$ and $d$ can be drawn as in Fig. 5. Notice that $d$ is the only control degree of freedom in the operation mode $A$ of UNI-AC; therefore, its voltage magnitude gain in this case can be described by a two-dimensional curve, where it is observed that the UNI-AC can offer bipolar voltage gain to either compensate the voltage sag or swell as a voltage stabilizer.

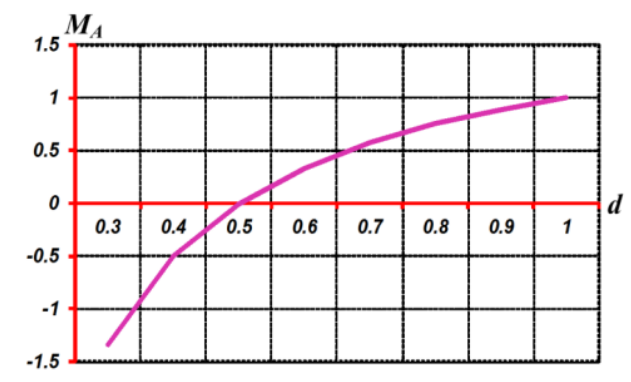

Fig. 5. Voltage amplitude transfer ratio of the UNI-AC under operation mode A using switching states I-II.

\section{B. Operation mode $B$}

This operation mode of the proposed UNI-AC employs the switching states I-III in Fig. 3 to offer a bipolar voltage transfer ratio. For positive voltage gain, $S_{4}$ is kept to be conducted while $S_{3}$ is turned off constantly; at the same time, $S_{1}$ and $S_{2}$ are modulated in a PWM manner. This scheme combines switching states I and III to form a normal buck type operation for the UNI-AC with a gain range from 0 to 1 . If negative voltage gain is required, states II and III are used, where $S_{I}$ remains blocked and $S_{2}$ is turned on as short circuit. Hence, the UNI-AC operates as a typical buck-boost converter with native voltage amplitude transfer ratio.

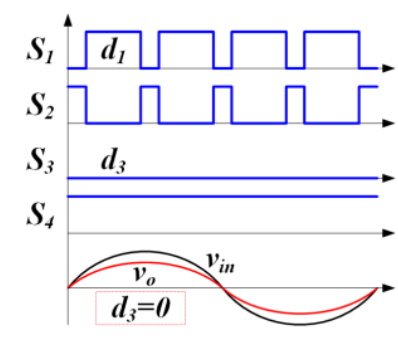

(a)

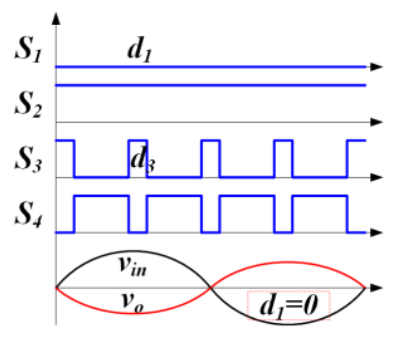

(b)
Fig. 6. Gate signals of the UNI-AC in operation mode B: (a) positive voltage gain; (b) negative voltage gain.

In mode $B$, the gate signals are shown in Fig. 6; also, the duty cycles of $S_{1}$ and $S_{3}, d_{1}$ and $d_{3}$, are used to describe the UNI-AC performance. Due to the hybrid buck and buck-boost operation, the dynamic equations of the UNI-AC in this case are piecewise, see (3) and (4). It is observed, when $d_{3}=0$, the UNI-AC works in buck mode; while if $d_{l}=0$, it is an inverting buck-boost converter. In further, by neglecting the variation of the state variables in (3) and (4) under steady state, the UNI-AC voltage gain in mode $B$ can be achieved as in (5) with bidirectional voltage output ability in a piecewise pattern.

$$
\left\{\begin{array}{l}
L \cdot \frac{d i_{L}}{d t}=d_{1} \cdot v_{i n}-v_{o} \\
C \cdot \frac{d v_{o}}{d t}=i_{L}-i_{o}
\end{array},\left\{\begin{array}{l}
M_{B} \in[0,1] \\
d_{3}=0
\end{array}\right.\right.
$$

$$
\begin{gathered}
\left\{\begin{array}{l}
L \cdot \frac{d i_{L}}{d t}=-d_{3} \cdot v_{i n}-\left(1-d_{3}\right) \cdot v_{o} \\
C \cdot \frac{d v_{o}}{d t}=-d_{3} \cdot i_{o}+\left(1-d_{3}\right) \cdot\left(i_{L}-i_{o}\right)
\end{array},\left\{\begin{array}{l}
M_{B}<0 \\
d_{1}=0
\end{array}\right.\right. \\
M_{B}=\frac{v_{o_{-} m}}{v_{\text {in_m }}}=\left\{\begin{array}{l}
d_{1}, \text { when } d_{3}=0 \\
-d_{3} /\left(1-d_{3}\right), \text { when } d_{1}=0
\end{array}\right.
\end{gathered}
$$

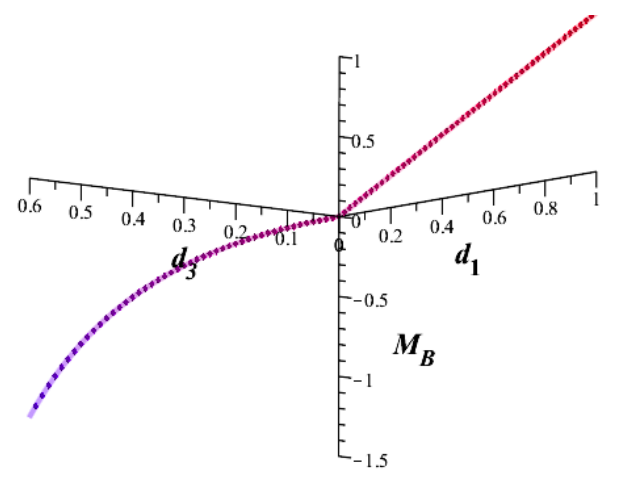

Fig. 7. Voltage amplitude transfer ratio of the UNI-AC under operation mode B using switching states I-III. 
From (5), the voltage gain of UNI-AC in mode $B$ can be displayed by Fig. 7 with a three-dimensional piecewise curve being produced. In the coordinates of $d_{1}, d_{3}$ and $M_{B}$, the first part of the space curve is distributed in the $d_{l}-M_{B}$ plane with $d_{3}=0$ for the buck operation; while the second piece is located in $d_{3}-M_{B}$ plane $\left(d_{l}=0\right)$ as a buck-boost converter for inverting voltage generation.

Also, mode $B$ offers lower switching losses for UNI-AC compared to mode $A$ due to its reduced total switching actions offered by the buck and buck-boost piecewise operation.

\section{Operation mode $C$}

The mode $C$ employs all switching states I-IV in Fig. 3 to supply maximum control degree of freedom for the UNI-AC. In this scenario, the duty cycles of both legs are in PWM manner as in Fig. 8, i.e. $d_{1}$ and $d_{3}$ are free variables that are coordinated to regulate the output voltage. Hence, the dynamic equations for UNI-AC mode $C$ is described by (6). Similarly, the voltage gain for this mode is manipulated to be (7).

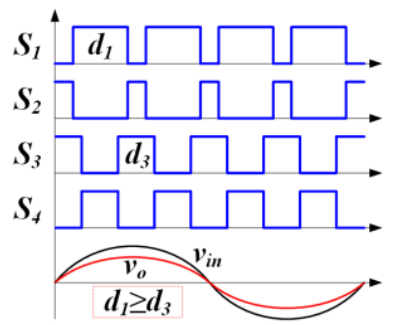

(a)

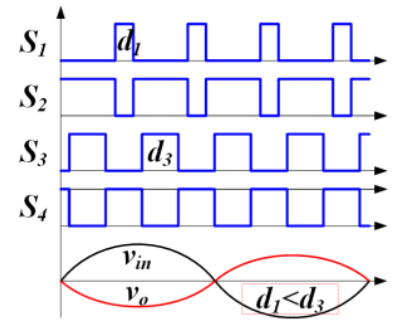

(b)
Fig. 8. Gate signals of the UNI-AC in operation mode C: (a) positive voltage gain; (b) negative voltage gain.

$$
\begin{aligned}
& \left\{\begin{array}{l}
L \cdot \frac{d i_{L}}{d t}=d_{1} \cdot v_{i n}-d_{3} \cdot v_{i n}-\left(1-d_{3}\right) \cdot v_{o} \\
C \cdot \frac{d v_{o}}{d t}=\left(1-d_{3}\right) \cdot i_{L}-i_{o}
\end{array}\right. \\
& M_{C}=\frac{v_{o_{-} m}}{v_{i n_{-} m}}=\frac{d_{1}-d_{3}}{1-d_{3}}
\end{aligned}
$$

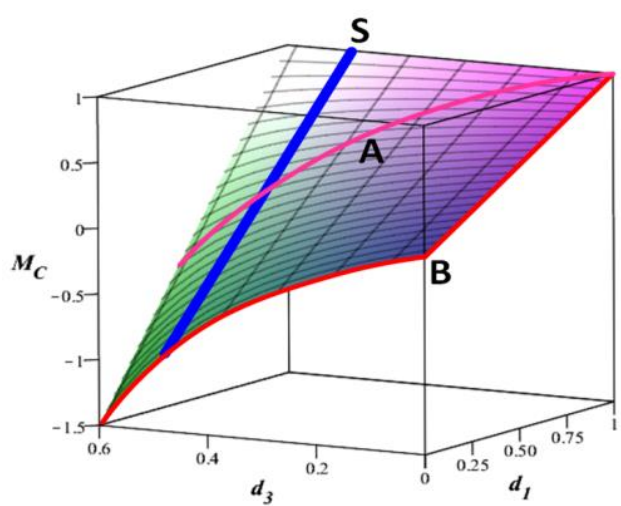

Fig. 9. Voltage amplitude transfer ratio of the UNI-AC under operation mode $\mathrm{C}$ using switching states I-IV.

In (7), since $d_{1}$ and $d_{3}$ are both free variables for the control of output voltage, the plot of $M_{c}$ depending on $d_{l}$ and $d_{3}$ is a three-dimensional surface as shown in Fig. 9. In this mode, to achieve a certain voltage gain for the UNI-AC, the two control inputs can have infinite number of combinations as observed from Fig. 9. In practical control design of the proposed UNI-AC in operation mode $C$, the signal of $d_{s}$ can be set to adjust the maximum inverting output voltage range and maintained constant when $d_{l}$ is changing for regulating the output voltage. In this manner, the linearity of the UNI-AC transfer function can be guaranteed. For example, in (7), if $d_{3}$ is set to be 0.5 , the voltage gain $M_{c}$ becomes a linear expression of $d_{1}$ ranging from -1 to 1 as shown by the line ' $\mathrm{S}$ ' in Fig. 9. In further, with different preset values of $d_{3}, M_{c}$ can be a family of straight lines in parallel with ' $\mathrm{S}$ ', resulting in the variant output voltage range. This method is an optimized way for indexing $d_{1}$ and $d_{3}$ from the three-dimensional surface of voltage gain in Fig. 9 when operating the UNI-AC under mode $C$.

It is worth noticing that the UNI-AC gain curves in mode $A$ and $B$ are both special cases of the voltage transfer ratio for mode $C$. When setting $d_{l}=1-d_{3}=d$ in (7), it is simplified to be the same as (2) for mode $A$; while if (7) is restricted by the assumption of either $d_{1}=0$ or $d_{3}=0$, the same conclusion as (5) for mode $B$ can be achieved. In Fig. 9, the identified curves 'A' and ' $B$ ' are transplanted version of the gain curves in Fig. 5 and Fig. 7 for operation mode $A$ and $B$, respectively.

\section{Performance Evaluation of UNI-AC}

In this section, the performance evaluation for the UNI-AC is carried out; and then, a comprehensive comparison between different operation modes of UNI-AC and other traditional ac-ac converters are presented.

\section{A. UNI-AC and current source ac-ac converter}

The conventional current source type ac-ac converter in [18] employs an intermediate current dc-link to connect the two back-to-back unidirectional switch based bridge circuits, where twice amount of switches are inserted in the conduction path compared to the UNI-AC, resulting in higher losses.

Another main drawback of this converter compared to UNI-AC is the increased size and weight due to the dc current carried by the intermediate inductor. While in the UNI-AC, the inductor current is in ac pattern with small footprint. Also, the common ground feature between input and output is lost in the current source ac-ac converter.

\section{B. UNI-AC and impedance source based bipolar ac-ac converters}

Observed from Fig. 3, the voltage stresses of $S_{1}$ and $S_{2}$ in UNI-AC are the same as the input voltage $v_{i n}$; while $S_{3}$ and $S_{4}$ sustain a voltage of $v_{i n}-v_{o}$. The current rating for all switches of UNI-AC are equal to the inductor current $i_{L}$.

By assuming the state variables variation rates in (1), (3), (4) and (6) to be zero, the steady state voltage transfer ratio of the UNI-AC under different modes have been achieved by (2), (5) and (7). Similarly, the transfer ratios $\left\{K_{A}, K_{B}, K_{C}\right\}$ for the inductor current magnitude $i_{L_{-} m}$ from load current magnitude $i_{o_{-} m}$ can be solved into (8), (9) and (10) for mode $A, B$ and $C$, respectively. Since mode $C$ is the generic case of UNI-AC, it is adopted for a comparison with the bipolar ac-ac converter in 
[14], the (modified) quasi-Z-source schemes in [11, 12], and the original Z-source ac-ac converters in $[9,10]$, see TABLE I.

$$
\begin{gathered}
K_{A}=\frac{i_{L_{-} m}}{i_{o_{-} m}}=\frac{1}{d} \\
K_{B}=\frac{i_{L_{-} m}}{i_{o_{-} m}}=\left\{\begin{array}{l}
1, \text { when } d_{3}=0 \\
1 /\left(1-d_{3}\right), \text { when } d_{1}=0
\end{array}\right. \\
K_{C}=\frac{i_{L_{-} m}}{i_{o_{-} m}}=\frac{1}{1-d_{3}}
\end{gathered}
$$

In this comparison, the fixed input voltage and load current magnitude $v_{i n \_m}$ and $i_{o_{-} m}$ are assumed for each converter; and based on (7) and (10), the voltage and current stresses of UNI-AC power switches under mode $C$ can be quantified as in TABLE I, where it is observed the proposed solution employs reduced number of passive components than the Z-source type converters, resulting in lower switching voltage and current ratings. Therefore, although more power switches are used in UNI-AC, its total semiconductor device cost and conduction losses are not increased compared to its rivals with high power rated switches in TABLE I. Besides, the overall size of UNI-AC is reduced drastically with the significant save on the passive elements, leading to a compact hardware design and a high power density. Also, the proposed converter is able to share a common ground for the input and output ports by the solid connection of the reference terminals.

Due to the increased control degree of freedom in the UNI-AC, its voltage gain is controlled by two variables, $d_{l}$ and $d_{3}$; while other candidates have only one control input. This can bring versatile modes of operation to the proposed converter as analyzed in section II with advanced features. For example, the dynamic response of UNI-AC is fast due to the less use of passive elements; further, in operation mode $B$ of the UNI-AC, the switching losses can be reduced significantly since there is only switching actions in one leg; also, the mode $C$ offers two independent control degrees of freedom in the UNI-AC with the possibility for a linear bipolar modulation range that is beneficial for the robustness and bandwidth of the controller due to the time-invariant features of the converter model. In contrast, the Z-source based converters usually have higher number of poles and zeros in their transfer functions including right-half-plane (RHP) zeros, which, as a time-variant model, will drift when the operation point changes in an ac system. This deteriorates the global stability and dynamic response of the ac-ac converter applied as a voltage compensator [16].

\section{Different operation modes of the UNI-AC}

In further, detailed performance of UNI-AC in different operation modes are investigated. If the desired voltage gain of the UNI-AC is from -1.5 to 1 , the switching voltage stresses are the same for all operation modes with $v_{i n \_m}$ (input voltage) for $\left\{S_{1}, S_{2}\right\}$ and $2.5 \times v_{\text {in_m }}$ (maximum difference between input and output voltages) for $\left\{S_{3}, S_{4}\right\}$; while the current ratings for the power switches under each mode are different depending on its extreme value of the duty cycle.

From (2), the control signal $d$ (duty cycle of $S_{l}$ ) should range from 0.2857 to 1 in operation mode $A$ to guarantee the desired output voltage range; similarly, using (5) for mode $B, d_{l}$ varies from 0 to 1 for non-inverting buck operation and $d_{3}$ changes from 0 to 0.6 for the inverting buck-boost voltage generation; in mode $C$, using the linear indexing method, $d_{3}$ is fixed as 0.6 and $d_{1}$ is modulated from 0 to 1 . Based on above observations and (8)-(10), the current stresses for the power devices of UNI-AC in each operation mode can be calculated as in TABLE II, where it is found mode $A$ suffers from the highest switching current stresses due to its higher duty cycle for $S_{3}$ than other cases for generating out-of-phase voltage.

From (1), the ripple voltage and ripple current on the state variables of the UNI-AC in mode $A$ can be estimated by (11), where $f_{s w}$ is the switching frequency. The maximum ripple components are then achieved as in TABLE II by substituting the minimum value of $d$ into (11) to generate the maximum out-of-phase voltage (inverting).

$$
\left\{\begin{array}{l}
\Delta i_{L_{-} A}=\frac{v_{i n_{-} m} \cdot(1-d)}{f_{s w} \cdot L} \\
\Delta v_{o_{-} A}=\frac{i_{o_{-} m} \cdot(1-d)}{f_{s w} \cdot C}
\end{array}\right.
$$

Based on (3) and (4) for mode $B$, the ripple components equations are in a piecewise pattern. Within positive gain area, the UNI-AC works in typical buck mode with minimum ripple components; thus, the ripple expressions in the inverting gain region are focused, see (12). As in TABLE II, the maximum ripples happen at the negative peak voltage gain point when $d_{3}$ reaches its maximum, which is 0.6 .

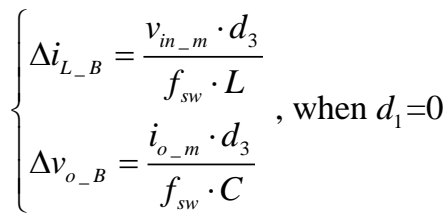

\begin{tabular}{|c|c|c|c|c|c|}
\hline & \multicolumn{2}{|c|}{ UNI-AC (in mode $C$ ) } & $\begin{array}{c}\text { Bipolar ac-ac converter in } \\
{[14]}\end{array}$ & $\begin{array}{c}\text { (modified) quasi-Z source } \\
\text { ac-ac converters in }[11,12]\end{array}$ & $\begin{array}{c}\text { Original Z-source ac-ac } \\
\text { converters in }[9,10]\end{array}$ \\
\hline Voltage gain & \multicolumn{2}{|r|}{$\frac{d_{1}-d_{3}}{1-d_{3}}$} & $\frac{2 d-1}{d}$ & $\frac{1-d}{1-2 d}$ & $\frac{1-d}{1-2 d}$ \\
\hline \multirow[b]{2}{*}{ Switching voltage stress } & $S_{1}, S_{2}$ & $v_{i n \_m}$ & \multirow{2}{*}{$v_{\text {in_m }} \times \frac{1}{d}$} & \multirow{2}{*}{$v_{i n_{-} m} \times \frac{1}{1-2 d}$} & \multirow{2}{*}{$v_{i n_{-} m} \times \frac{1}{1-2 d}$} \\
\hline & $S_{3}, S_{4}$ & $v_{i n_{-} m} \times \frac{1-d_{1}}{1-d_{3}}$ & & & \\
\hline Switching current stress & \multicolumn{2}{|c|}{$i_{o_{-} m} \times \frac{1}{1-d_{3}}$} & $i_{o_{-} m} \times \frac{1}{d}$ & $i_{o_{-} m} \times \frac{1}{1-2 d}$ & $i_{o_{-} m} \times \frac{1}{1-2 d}$ \\
\hline Passive device & \multicolumn{2}{|c|}{ one inductor, one capacitor } & $\begin{array}{l}\text { two inductors, two } \\
\text { capacitors }\end{array}$ & $\begin{array}{l}\text { three or two inductors, three } \\
\text { or two capacitors }\end{array}$ & $\begin{array}{l}\text { three inductors, three } \\
\text { capacitors }\end{array}$ \\
\hline Ground sharing & & Yes & Yes & Yes & Possible \\
\hline
\end{tabular}

In mode $C$, from Fig.7 and (7), when $d_{1}$ is larger than $d_{3}$ for

TABLE I

Comparison Results Between the Proposed UNI-AC and Representative Z-Source Type AC-AC Converters 
positive voltage gain, the switching state II in Fig. 3 will not be used; while if $d_{1}$ is smaller than $d_{3}$ for negative voltage transfer ratio, the switching state I does not emerge. Similarly, in this mode, the UNI-AC ripple performance becomes worse during the voltage inverting region; hence, (13) is employed to calculate the maximum ripple current and voltage, which are shown in TABLE II with $d_{3}=0.6$ and $d_{1}=0$.

$$
\left\{\begin{array}{l}
\Delta i_{L_{-} C}=\frac{v_{i n_{-} m} \cdot\left(d_{3}-d_{1}\right)}{f_{s w} \cdot L} \\
\Delta v_{o_{-} C}=\frac{i_{o_{-} m} \cdot\left(d_{3}-d_{1}\right)}{f_{s w} \cdot C}
\end{array}, \text { when } d_{1}<d_{3}\right.
$$

TABLE II

COMPARISON BETWEEN EACH OPERATION MODES OF UNI-AC WITH VOLTAGE GAIN FROM - 1.5 TO 1 AND FIXED LOAD CURRENT I I-M

\begin{tabular}{|c|c|c|c|}
\hline $\begin{array}{c}\text { UNI-AC } \\
\text { operation mode }\end{array}$ & Mode $A$ & Mode $B$ & Mode $C$ \\
\hline $\begin{array}{c}\text { Switching } \\
\text { current rating }\end{array}$ & $3.5 \times i_{o_{-} m}$ & $2.5 \times i_{o_{-} m}$ & $2.5 \times i_{o_{-} m}$ \\
\hline $\begin{array}{c}\text { Maximum } \\
\text { voltage ripple }\end{array}$ & $0.7143 \times \frac{i_{o_{\_} m}}{f_{s w} C}$ & $0.6 \times \frac{i_{o_{-} m}}{f_{s w} C}$ & $0.6 \times \frac{i_{o_{-} m}}{f_{s w} C}$ \\
\hline $\begin{array}{c}\text { Maximum } \\
\text { current ripple }\end{array}$ & $0.7143 \times \frac{v_{i n \_m}}{f_{s w} L}$ & $0.6 \times \frac{v_{i n \_m}}{f_{s w} L}$ & $0.6 \times \frac{v_{i n \_} m}{f_{s w} L}$ \\
\hline Conduction loss & Relatively high & Low & High \\
\hline Switching loss & Relatively high & Low & Relatively high \\
\hline $\begin{array}{c}\text { Control degree } \\
\text { of freedom }\end{array}$ & One & One & Two \\
\hline
\end{tabular}

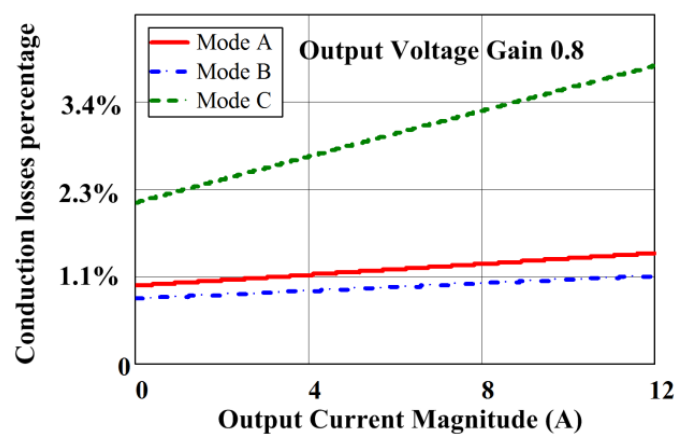

(a)

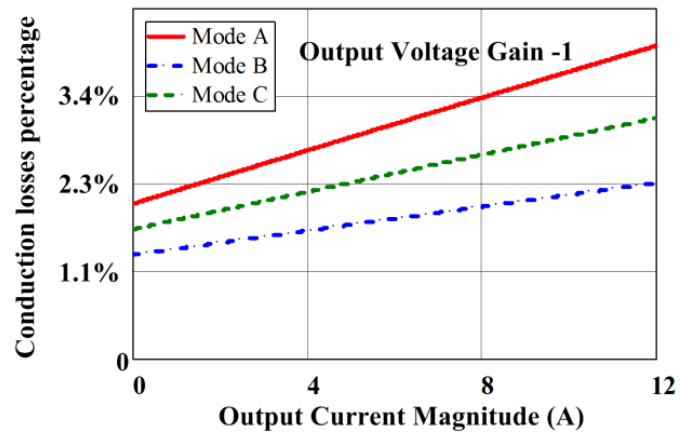

(b)

Fig. 10. Percentage of the power switch conduction losses in the UNI-AC: (a) output voltage gain is 0.8 ; (b) output voltage gain is -1 .

To evaluate the conduction losses of power semiconductor devices in the proposed UNI-AC, two operational points with bipolar voltage transfer ratio of 0.8 and -1 are examined for each mode. It is observed from Fig. 3, in each instance, there are always two switches from different legs in the conduction path. Since the voltage ratings of power switches are the same for each mode, the equivalent IGBT parameters including their total forward voltage drop and equivalent resistance are fixed and denoted by $V_{F}$ and $R_{a v}$, respectively. Thus, if the output current is expressed by (14), from (10), the generic conduction losses are obtained by (15). For given output voltage $v_{o_{-} m}$, the output power $S_{o}$ is equal to $1 / 2 v_{o_{-} m} \times i_{o_{-} m}$; and the percentage of semiconductor conduction losses are calculated using (16).

$$
\begin{gathered}
i_{o}=i_{o_{-} m} \cdot \sin \omega t \\
P_{c o n}=\frac{1}{\pi} \int_{0}^{\pi}\left(V_{F} \cdot i_{L}+R_{a v} \cdot i_{L}^{2}\right) \cdot d \omega t \\
=\frac{2 V_{F}}{\pi} \cdot \frac{i_{o_{-} m}}{1-d_{3}}+R_{a v} \cdot \frac{i_{o_{-} m}{ }^{2}}{2\left(1-d_{3}\right)^{2}} \\
\lambda=\frac{P_{c o n}}{S_{o}+P_{c o n}}
\end{gathered}
$$

Specifically, the equivalent duty cycle of $d_{3}$ for two cases with 0.8 and -1 voltage gains can be determined using (2), (5) and (7). Then, assuming $v_{\text {in_m } m}=150 \mathrm{~V}, V_{F}=0.8 \mathrm{~V}$ and $R_{a v}=0.03 \Omega$, the conduction losses in percentage for the above cases can be plotted in Fig. 10, where mode $B$ has the lowest on-state power dissipations. Also, as stated previously, mode $B$ can reduce the switching losses with the decreased switching actions.

The above conclusions are listed in TABLE II, where it is concluded that mode $B$ is an optimized operational trajectory for the UNI-AC in terms of efficiency performance; while the mode $C$ offers superior control flexibility.

\section{DESIGN OF THE UNI-AC}

Based on previous analysis, the guidelines of the parameter selection for the UNI-AC are provided using a scale-down case study in following procedure:

- The known parameters: input peak voltage $v_{\text {in_m }}=150 \mathrm{~V}$, output voltage gain range $\left[M_{n}\right.$, $\left.M_{p}\right]=[-1.5,1]$, total load impedance $Z_{L}=40 \Omega$, switching frequency $f_{s w}=25 \mathrm{kHz}$, allowable current and voltage ripples $\kappa_{i}=20 \%$ of $i_{L_{-} m}$ and $\kappa_{v}=10 \%$ of $v_{o \_m}\left(\right.$ or $\left.v_{\text {in_m }}\right)$;

- $\quad$ Recall Fig. 2, voltage stresses of $S_{1}$ and $S_{2}$ are equal to $v_{i n \_m}=150 \mathrm{~V}$; the voltage stresses of $S_{3}$ and $S_{4}$ are the subtraction of $v_{\text {in } \_m}$ and the maximum inverting output voltage, which is expressed as $v_{i n \_m} \times\left(1-M_{n}\right)$ $=375 \mathrm{~V}$;

- $\quad$ Based on (2), (5) and (7), the duty cycle of $S_{3}$ for generating the maximum out-of-phase voltage (the voltage gain $M_{n}$ ) in each mode can be obtained as (17);

$$
\left\{\begin{array}{l}
d_{3_{-} A}^{\max }=1-1 /\left(2-M_{n}\right)=0.7143 \\
d_{3_{-} B}^{\max }=1-1 /\left(1-M_{n}\right)=0.6 \\
d_{3_{-} C}^{\max }=0.6
\end{array}\right.
$$

- With maximum load current in (18); the switch current stresses in each mode can be calculated by (19);

$$
i_{o_{-} m}^{\max }=\frac{v_{i n_{-} m} \cdot \max \left(\left|M_{n}\right|,\left|M_{p}\right|\right)}{Z_{L}} \approx 5.6 \mathrm{~A}
$$




$$
\left\{\begin{array}{l}
I_{s t r_{-} A}=i_{o_{-} m}^{\max } /\left(1-d_{3_{-} A}^{\max }\right) \approx 20 \mathrm{~A} \\
I_{s t r_{-} B}=i_{o_{-} m}^{\max } /\left(1-d_{3_{-} B}^{\max }\right) \approx 14 \mathrm{~A} \\
I_{s t r_{-} C}=i_{o_{-} m}^{\max } /\left(1-d_{3_{-} C}^{\max }\right) \approx 14 \mathrm{~A}
\end{array}\right.
$$

- The inductance value can be determined by (20), based on (11)-(13);

$$
\left\{\begin{array}{l}
L_{A}^{\min }=\frac{v_{i n \__{-}} \cdot d_{3_{-} A}^{\max } \cdot\left(1-d_{3_{-} A}^{\max }\right)}{f_{s w} \cdot \kappa_{i} \cdot i_{o_{-} m}^{\max }}=1.09 \mathrm{mH} \\
L_{B}^{\min }=\frac{v_{\text {in } m} \cdot d_{3_{-} B}^{\max } \cdot\left(1-d_{3_{-} B}^{\max }\right)}{f_{s w} \cdot \kappa_{i} \cdot i_{o_{-} m}^{\max }}=1.28 \mathrm{mH} \\
L_{C}^{\min }=\frac{v_{i n_{-} m} \cdot d_{3_{-} C}^{\max } \cdot\left(1-d_{3_{-} C}^{\max }\right)}{f_{s w} \cdot \kappa_{i} \cdot i_{o_{-} m}^{\max }}=1.28 \mathrm{mH}
\end{array}\right.
$$

- The required input capacitance depends on the duration of current discontinuity in the input, which is nearly zero for mode $A$. For other modes, with the maximum fundamental input current in (21); the needed minimum capacitance can be calculated based on (22);

$$
\begin{gathered}
i_{i n_{-} m}^{\max }=\frac{v_{i n \__{-} m} \cdot M_{n}^{2}}{Z_{L}} \approx 8.44 \mathrm{~A} \\
C_{i n}^{\min }=\frac{i_{i n_{-} m}^{\max } \cdot\left(1-d_{3_{-} B}^{\max }\right)}{\kappa_{v} \cdot v_{i n_{-} m} \cdot f_{s w}}=9 \mu \mathrm{F}
\end{gathered}
$$

- The output capacitance is chosen by (23) based on the voltage ripple requirement.

$$
\left\{\begin{array}{l}
C_{A}^{\min }=\frac{i_{o_{-} m}^{\max } \cdot d_{3_{-} A}^{\max }}{f_{s w} \cdot \kappa_{v} \cdot v_{i n_{-} m} \cdot\left|M_{n}\right|}=7.15 \mu \mathrm{F} \\
C_{B}^{\min }=\frac{i_{o_{-} m}^{\max } \cdot d_{3_{-} B}^{\max }}{f_{s w} \cdot \kappa_{v} \cdot v_{i n_{-} m} \cdot\left|M_{n}\right|}=6 \mu \mathrm{F} \\
C_{C}^{\min }=\frac{i_{o_{-} m}^{\max } \cdot d_{3_{-} C}^{\max }}{f_{s w} \cdot \kappa_{v} \cdot v_{i n_{-} m} \cdot\left|M_{n}\right|}=6 \mu \mathrm{F}
\end{array}\right.
$$

- For all above calculated passive devices, their reactive power ratings should be reexamined as for an ac system, which are required be within the reasonable range of the total power capacity.

To be compatible with all operation modes, the experiment specifications of UNI-AC are selected as in TABLE III, where MOSFETs and diodes are adopted as bidirectional switches in this low voltage design case.

TABLE III

SPECIFICATION OF THE UNI-AC EXPERIMENT

\begin{tabular}{cc}
\hline \hline Input voltage peak value $v_{i n_{-} m}$ & $150 \mathrm{~V}$ \\
Load impedance $Z_{L}$ & $40 \Omega$ \\
Power rating & $300 \mathrm{VA}$ \\
Switching frequency $f_{s w}$ & $25 \mathrm{kHz}$ \\
Inductance $L$ & $1.3 \mathrm{mH}$ \\
Output capacitance C & $10 \mu \mathrm{F}$ \\
Input capacitance $C_{i n}$ & $10 \mu \mathrm{F}$ \\
Bidirectional switch $S_{1}-S_{4}$ & SPHX0N60S5 and STTH6012 \\
\hline \hline
\end{tabular}

In fact, the right terms of the volt-second balance equations in (1), (3), (4) and (6) are not zero in steady state due to the reactive power consumption of inductors; instead, they should be equal to the loop voltage drop caused by inductor current $i_{L}$ flowing the inner impedance $r$ (loop resistance) and $\omega L$ ( $\omega$ is the fundamental angular frequency). If the load impedance is $Z_{L}$ and $\lambda$ is impedance ratio in (24), the voltage gains of UNI-AC in each mode can be revised as (25), (26) and (27).

$$
\begin{gathered}
\lambda=\frac{\sqrt{\omega^{2} L^{2}+r^{2}}}{Z_{L}} \\
M_{A}^{*}=\frac{v_{o_{-} m}}{v_{i n_{-} m}}=\frac{(2 d-1) \cdot d}{d^{2}+\lambda} \\
M_{B}^{*}=\frac{v_{o_{-} m}}{v_{\text {in_m }}}=\left\{\begin{array}{l}
\frac{d_{1}}{1+\lambda}, \text { when } d_{3}=0 ; \\
-\frac{d_{3} \cdot\left(1-d_{3}\right)}{\left(1-d_{3}\right)^{2}+\lambda}, \text { when } d_{1}=0 . \\
M_{C}^{*}=\frac{v_{o_{-} m}}{v_{\text {in_m }}}=\frac{\left(d_{1}-d_{3}\right) \cdot\left(1-d_{3}\right)}{\left(1-d_{3}\right)^{2}+\lambda}
\end{array}\right.
\end{gathered}
$$

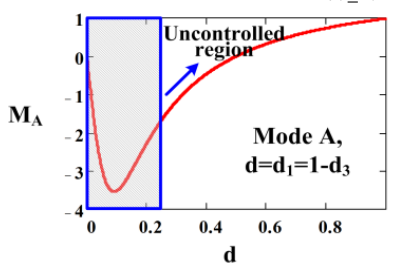

(a)

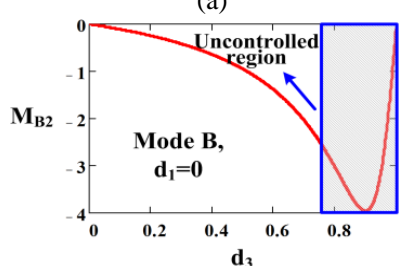

(c)

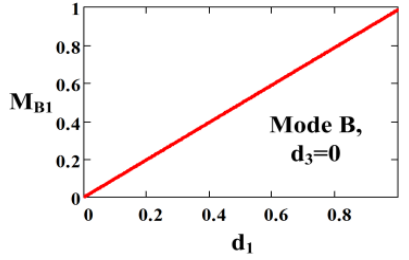

(b)

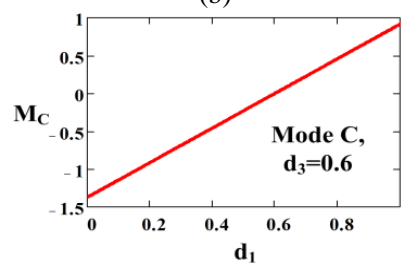

(d)
Fig. 11. Practical gains of UNI-AC considering inner impedance: (a) mode A; (b) mode B (positive); (c) mode B (negative); (d) mode C.

With the parameters in TABLE III and the total conduction path resistance of $0.45 \Omega$, the voltage gains in (25), (26) and (27) can be plotted by Fig. 11. It is observed from Fig. 11(a) and (c), when the duty cycle of $S_{3}$ is larger than a critical value, the output voltage gain will decrease significantly due to its inner voltage drop, which is similar as in normal boost dc-dc converter. This has resulted in an uncontrollable region for the proposed UNI-AC. Hence, in the test case of TABLE III, $d_{3}$ is limited to be lower than 0.75 .

\section{SIMULATION AND EXPERIMENT}

\section{A. Simulation Results}

The simulation results of the UNI-AC with specifications in TABLE III are shown in Fig. 12. In this test, the output voltage is controlled as $120 \mathrm{~V}$ in-phase in Fig. 12(a) with the largest inductor current in mode $C$; then, the output voltage is set to be $150 \mathrm{~V}$ out-of-phase in Fig. 12(b); and inductor current in mode $A$ is largest. This observation agrees with previous analysis.

Furthermore, the sampled waveforms of voltage across the bidirectional switches $S_{2}$ and $S_{4}$ with both positive and negative voltage gains are given for operation mode $C$ in Fig. 12(c) and (d), respectively. 


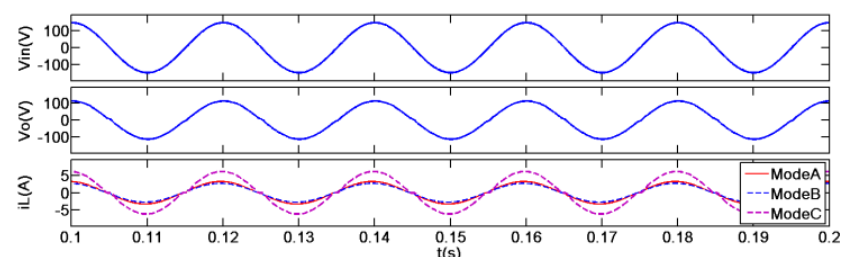

(a)

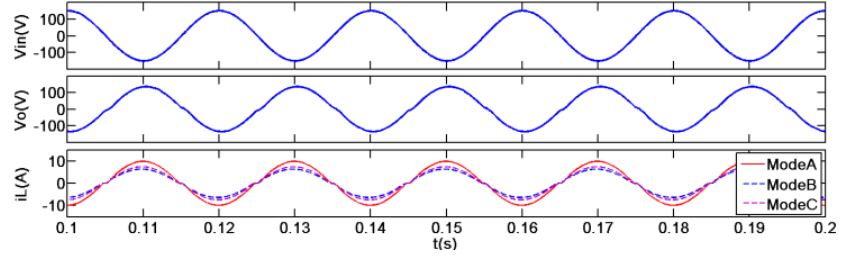

(b)

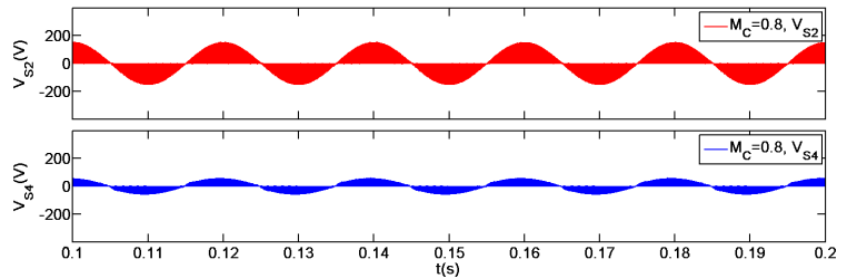

(c)

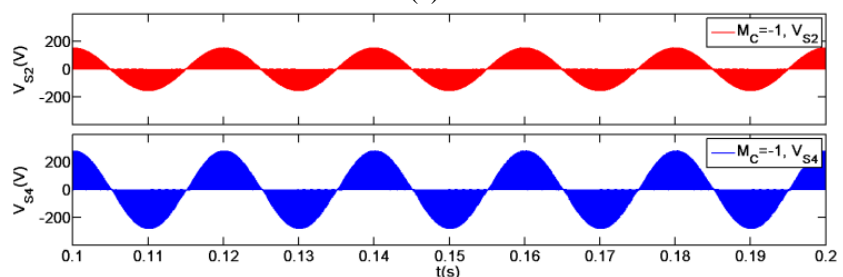

(d)

Fig. 12. Simulation results for the UNI-AC: (a) $120 \mathrm{~V}$ non-inverting output voltage and inductor current; (b) $150 \mathrm{~V}$ inverting output voltage and inductor current; (c) voltage of switches $S_{2}$ and $S_{4}$ with in-phase output voltage under mode C; (d) voltage of switches $S_{2}$ and $S_{4}$ with out-of-phase output voltage under mode C.

\section{B. Experiment Verification}

For further verifications, a prototype of the UNI-AC has been built and tested using the same specifications in TABLE III; then, an Infineon TC1796 DSP platform is adopted for the digital modulation of the proposed converter. The photo of the experimental setup is displayed in Fig. 13.

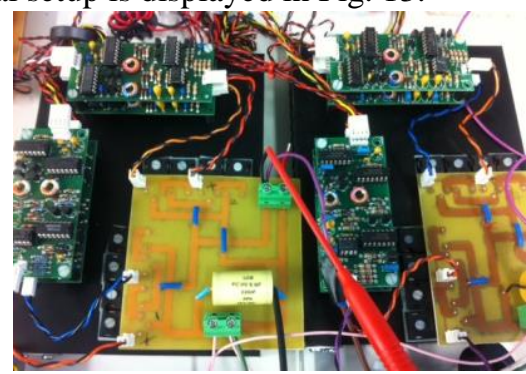

Fig. 13. Photo of the experimental setup.

The UNI-AC test rig works in operation mode $A, B$ and $C$ at pre-set output voltage gain of 0.8 and -1 , respectively. In the PWM implementation, all the gate signals are arranged in the centre-aligned pattern. Specially, the duty cycle $d_{3}$ is set to 0.6 for operation mode $C$. The safe commutation strategy in [13,
19] is adopted to avoid additional passive snubber circuits.

Fig. 14 shows the voltage waveforms of the bidirectional switches $S_{2}$ and $S_{4}$ for each case. It is observed that when the voltage gain of the UNI-AC changes to negative direction, for all the operation modes, the switching voltage stresses on the switches $S_{3}$ and $S_{4}$ become larger than those in positive gain region. This is because the switch leg composed by $S_{1}$ and $S_{2}$ sustains a total voltage equal to input voltage; while the $S_{3}$ and $S_{4}$ together have to block the voltage difference between the input and output terminals. Specially, minimized switching action (thus, switching losses) can be achieved by the operation mode $B$ of the proposed converter as in Fig. 14(b) and (c).

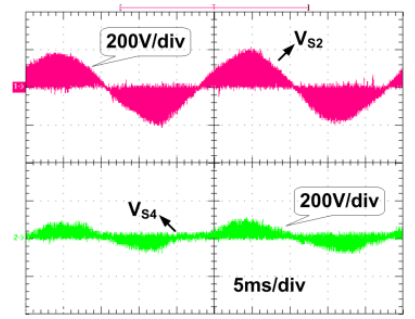

(a)

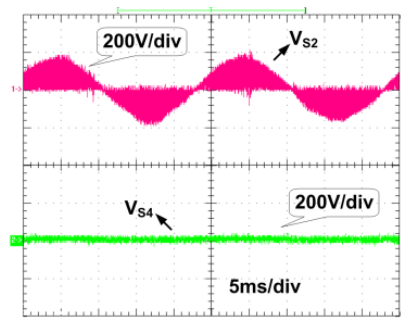

(c)

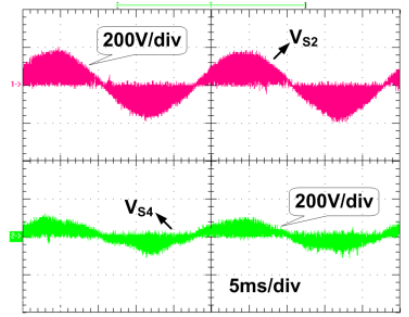

(e)

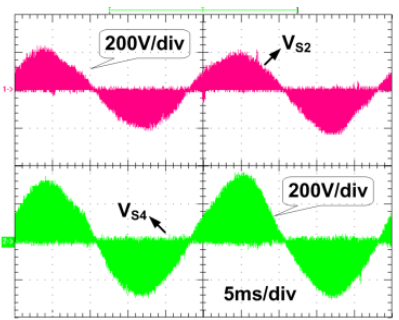

(b)

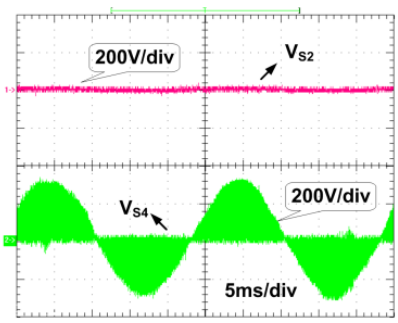

(d)

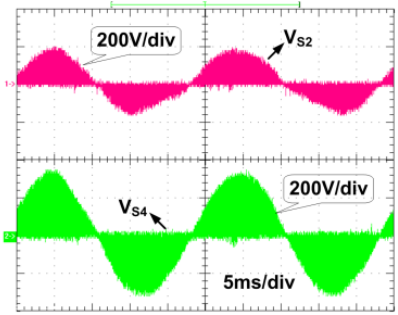

(f)
Fig. 14. Voltage on the bidirectional switches (Vs2 and Vs4): (a) mode A with voltage gain of 0.8 ; (b) mode $A$ with voltage gain of -1 ; (c) mode $B$ with voltage gain of 0.8 ; (d) mode $B$ with voltage gain of -1 ; (e) mode $C$ with voltage gain of 0.8 ; (f) mode $C$ with voltage gain of -1 .

The line frequency waveforms for UNI-AC are also shown in Fig. 15, where the input voltage $v_{i n}$, inductor current $i_{L}$ and output voltage $v_{o}$ are displayed for the two pre-set operation points under each mode, respectively. In positive gain region, operation mode $C$ has the highest current stress for the power switches (inductor current) due to the large conduction period of $S_{3}$, which results in poorest efficiency performance in this region. On the other side, within the negative voltage gain area, the average switching current for mode $A$ increases and finally exceeds that in mode $C$. Hence, mode $A$ is not competitive for producing high magnitude out-of-phase voltage compared to mode $B$ and $C$ due to the relatively large circulating current stimulated through the power switches. Among all operation cases, mode $B$ offers the lowest average current and conduction 
losses for the power switches. These observations are in line with the analysis and conclusions in TABLE II.

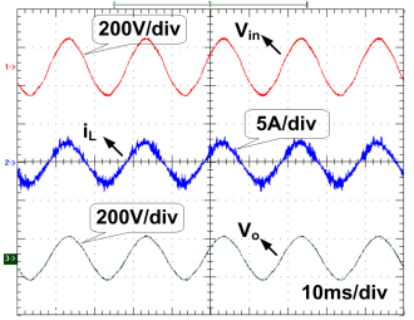

(a)

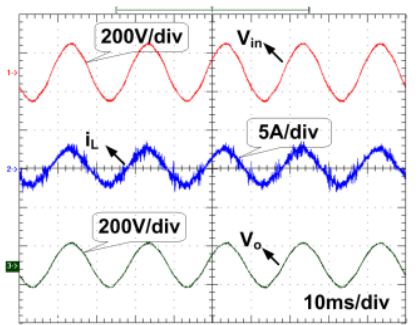

(c)

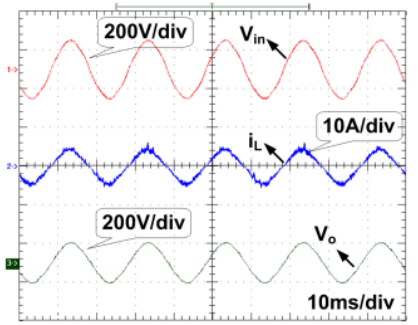

(e)

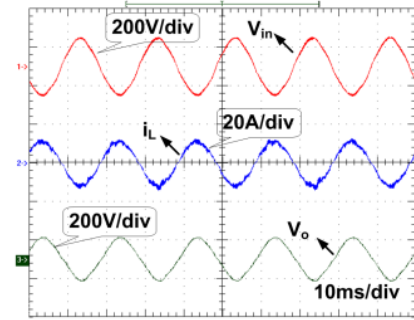

(b)

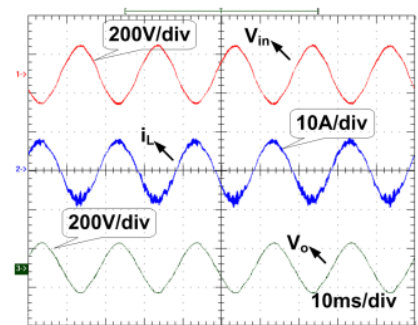

(d)

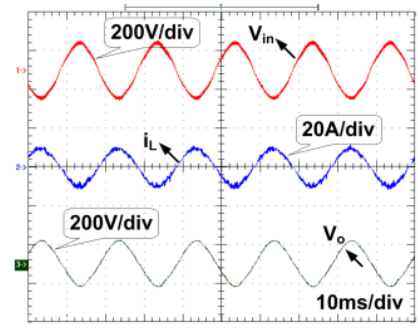

(f)
Fig. 15. The input voltage (vin), output voltage (vo) and inductor current (iL) waveforms: (a) mode $A$ with voltage gain of 0.8 ; (b) mode $A$ with voltage gain of -1 ; (c) mode $B$ with voltage gain of 0.8 ; (d) mode $B$ with voltage gain of -1 ; (e) mode $C$ with voltage gain of 0.8 ; (f) mode $C$ with voltage gain of -1 .

The measured total harmonic distortion (THD) values of the UNI-AC output voltage with -1 voltage gain for each operation mode are listed in TABLE IV. As is analysed previously, the switch voltage stresses of the UNI-AC are much lower than those in the impedance network based converters, which means the overall $d v / d t$ is smaller. Thus, generally, a reduced THD in the UNI-AC can be expected. Further, different modes of the UNI-AC have different PWM patterns and harmonic content. The use of zero voltage states in mode $B$ reduces the total number of switching instances and achieves lowest THD.

TABLE IV

MEASURED UNI-AC OUTPUT VOLTAGE THD IN EACH MODE

\begin{tabular}{cccc}
\hline \hline & Mode $A$ & Mode $B$ & Mode $C$ \\
$\begin{array}{c}\text { UNI-AC output } \\
\text { voltage THD }\end{array}$ & $3.56 \%$ & $3.34 \%$ & $3.88 \%$ \\
\hline \hline
\end{tabular}

If the input voltage is $150 \mathrm{~V}$ (peak value) and the nominal resistive load is $R_{L}=40 \Omega$, by varying the load $R$, the UNI-AC efficiency for mode $A, B$ and $C$ at voltage gain of 0.8 and -1 are shown in Fig. 16, which indicates that mode $B$ has highest efficiency performance for both 0.8 and -1 voltage gains. This is achieved by the reduced average current in the power device and the minimized switching actions. The efficiency for mode
$A$ changes drastically for different operational points since the duty cycle $d_{3}$ (thus, average current in the power switches) has the largest variation range among all modes. While in mode $C$, since $d_{3}$ is fixed to offer a linear modulation range as shown in Fig. 11(d), the conduction losses of the UNI-AC in this case are less influenced by the voltage gain change; thus, its overall efficiency difference at different operational points are mainly determined by the switching losses.

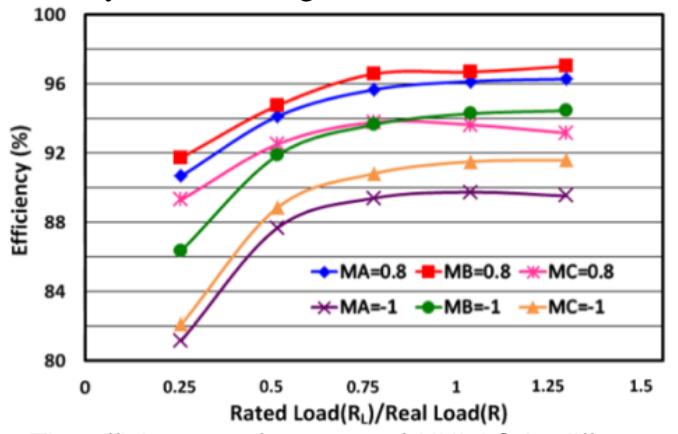

Fig. 16. The efficiency performance of UNI-AC in different modes and operation points with load variations.

\section{Simulation Study of UNI-AC Based DVR}

Simulation of the UNI-AC applied as a DVR is carried out based on the configuration of Fig. 1. The nominal grid phase voltage is $240 \mathrm{~V}$ (root-mean-square, RMS). In the UNI-AC device, $10 \mu \mathrm{H}$ inductor and $10 \mu \mathrm{F}$ output capacitor are adopted. The system power rating is $10 \mathrm{kVA}$ per phase.

Fig. 17 shows that the UNI-AC is able to generate either in-phase or out-of-phase voltage to compensate both voltage sag and swell problems. During 0s 0.1s, the grid remains stable, and the UNI-AC device produces dominantly reactive power to support the grid voltage at the load bus. Then, at $0.1 \mathrm{~s}$, the grid voltage starts a $20 \%$ swell and the UNI-AC generates out-of-phase voltage to force the voltage at the critical bus to track the reference. Finally, at $0.2 \mathrm{~s}$, the grid experiences $30 \%$ sag, which is managed by the UNI-AC with in-phase voltage injection.

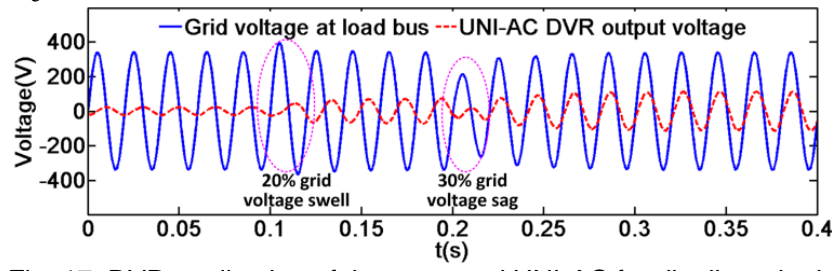

Fig. 17. DVR application of the proposed UNI-AC for distributed grid voltage sag/swell compensation.

\section{CONCLUSION}

In this paper, the unified non-inverting and inverting PWM ac-ac converter (UNI-AC) with reduced passive components and high power density is analysed. Thanks to its increased control degrees of freedom, versatile modes of operation can be established for the UNI-AC. Further, the law of operation and the performance analysis for each mode are presented in details. Mode $A$ has the simplest modulation scheme with only two switching states being used; however, its power switch 
current stresses, conduction losses and ripple performances are uncompetitive compared to other operation modes. Mode $B$ is able to reduce the losses significantly due to its lower current stresses for switches and the decreased total switching actions. Mode $C$ offers maximum control flexibility with both two legs being modulated independently (higher switching losses than mode $B$ ). By certain arrangement for the modulating signals, this mode is able to manipulate the converter average model to be a linear form with enhanced global stability and robustness. The UNI-AC also has a common ground shared by the input and output ports. Experimental work has been carried out to verify the feasibility and effectiveness of the UNI-AC.

\section{REFERENCES}

[1] F. C. L. Trindade, K. V. d. Nascimento, and J. C. M. Vieira, "Investigation on Voltage Sags Caused by DG Anti-Islanding Protection," IEEE Trans. Power Del., vol. 28, pp. 972-980, Apr., 2013.

[2] J. Y. Chan and J. V. Milanovic, "Assessment of the Economic Value of Voltage Sag Mitigation Devices to Sensitive Industrial Plants," IEEE Trans. Power Del., vol. 30, no. 6, pp. 2374-2382, Dec 2015.

[3] Y. W. Li, D. M. Vilathgamuwa, F. Blaabjerg, and P. C. Loh, "A Robust Control Scheme for Medium-Voltage-Level DVR Implementation," IEEE Trans. Ind. Electron., vol. 54, pp. 2249-2261, Aug., 2007.

[4] D. Vilathgamuwa, H. M. Wijekoon, and S. S. Choi, "A Novel Technique to Compensate Voltage Sags in Multiline Distribution System - The Interline Dynamic Voltage Restorer," IEEE Trans. Ind. Electron., vol. 53, pp. 1603-1611, Oct., 2006.

[5] A. M. Rauf and V. Khadkikar, "An Enhanced Voltage Sag Compensation Scheme for Dynamic Voltage Restorer," IEEE Trans. Ind. Electron., vol. 62, pp. 2683-2692, May, 2015.

[6] S. Jothibasu and M. K. Mishra, "An Improved Direct AC-AC Converter for Voltage Sag Mitigation," IEEE Trans. Ind. Electron., vol. 62, pp. 21-29, Jan., 2015.

[7] S. Jothibasu and M. K. Mishra, "A Control Scheme for Storageless DVR Based on Characterization of Voltage Sags," IEEE Trans. Power Del., vol. 29, pp. 2261-2269, Jul., 2014.

[8] J. Kaniewski, Z. Fedyczak, and G. Benysek, "AC Voltage Sag/Swell Compensator Based on Three-Phase Hybrid Transformer With Buck-Boost Matrix-Reactance Chopper," IEEE Trans. Ind. Electron., vol. 61, pp. 3835-3846, Aug., 2014.

[9] F. Xu Peng, Q. Zhao Ming, and P. Fang Zheng, "Single-phase Z-source PWM AC-AC converters," IEEE Power Electron. Letters, vol. 3, pp. 121-124, Dec., 2005.

[10] S. X. Y. Tang, and C. Zhang,, "Z-Source AC-AC Converters Solving Commutation Problem," IEEE Trans. Power Electron., vol. 22, pp. 2146-2154, Nov., 2007.

[11] N. Minh-Khai, J. Young-Gook, and L. Young-cheol, "Single-phase AC/AC converter based on quasi-Z-source topology," in Proc. IEEE Int. Symp. Ind. Electron., 2009, pp. 261-265.

[12] M. K. Nguyen, Y. C. Lim, and Y. J. Kim, "A Modified Single-Phase Quasi-Z-Source AC-AC Converter," IEEE Trans. Power Electron., vol. 27, no. 1, pp. 201-210, Jan 2012.

[13] R. A. M. R. Banaei, N. Jahanyari, and E. S. Najmi,, "An AC Z-Source Converter Based on Gamma Structure With Safe-Commutation Strategy," IEEE Trans. Power Electron., vol. 31, pp. 1255-1262, Feb., 2016.

[14] Z. Fedyczak, L. Frackowiak, M. Jankowski, and A. Kempski, "Single-phase serial AC voltage controller based on bipolar PWM AC matrix-reactance chopper," in Proc. Eur. Conf. Power Electron. Appl., 2005, pp. 1-10.

[15] F. Ahmed, Cha, H., Khan, A. and Kim, H.G.,, "A Novel Buck-Boost AC-AC Converter With Both Inverting and Noninverting Operations and Without Commutation Problem," IEEE Trans. Power Electron., vol. 31, pp. 4241-4251, Jun., 2016

[16] P. Li, G. P. Adam, Y. Hu, D. Holliday, and B. W. Williams, "Three-Phase AC-Side Voltage-Doubling High Power Density Voltage Source Converter With Intrinsic Buck-Boost Cell and Common-Mode Voltage Suppression," IEEE Trans. Power Electron., vol. 30, pp. 5284-5298, Sep., 2015.
[17] S. Jian and H. Grotstollen, "Symbolic analysis methods for averaged modeling of switching power converters," IEEE Trans. Power Electron., vol. 12, pp. 537-546, May, 1997.

[18] P. N. Enjeti, P. D. Ziogas, and J. F. Lindsay, "A current source PWM inverter with instantaneous current control capability," IEEE Trans. Ind. Appl., vol. 27, pp. 582-588, May/Jun., 1991.

[19] P. Li, Y. Wang, G. Philip Adam, D. Holliday, and B. W. Williams, "Three-Phase AC-AC Hexagonal Chopper System With Heterodyne Modulation for Power Flow Control Enhancement," IEEE Trans. Power Electron., vol. 30, pp. 5508-5521, Oct., 2015.

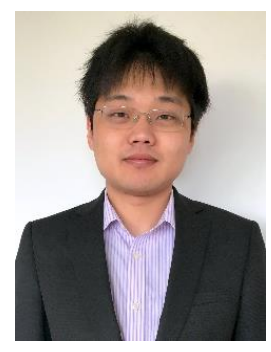

Peng Li (M'16) received the B.Sc. and M.Sc. degree both from the Department of Electrical Engineering, Zhejiang University, Hangzhou, China, in 2009 and 2012, respectively. In 2015, he received the Ph.D. degree from Electronic \& Electrical Engineering Department, University of Strathclyde, Glasgow, U.K., where he is currently working as a postdoctoral research fellow.

His research interests include high capacity power converters and the networking of power electronics units for power system applications.

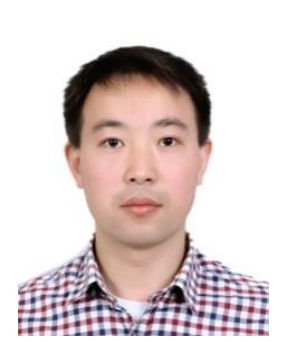

Yihua Hu (M'13-SM'15) received the B.S. degree in electrical motor drives in 2003, and the Ph.D. degree in power electronics and drives in 2011, both from China University of Mining and Technology, Jiangsu, China. Between 2011 and 2013, he was with the College of Electrical Engineering, Zhejiang University as a Postdoctoral Fellow. Between November 2012 and February 2013, he was an academic visiting scholar with the School of Electrical and Electronic Engineering, Newcastle University, Newcastle upon Tyne, UK. Between 2013 and 2015, he worked as a Research Associate at the power electronics and motor drive group, the University of Strathclyde. Currently, he is a Lecturer at the Department of Electrical Engineering and Electronics, University of Liverpool (UoL). He has published more than 50 peer reviewed technical papers in leading journals. His research interests include PV generation system, power electronics converters \& control, and electrical motor drives. 